\title{
Multidisciplinary approach for developing a new robotic system for domiciliary assistance to elderly people
}

\author{
F. Cavallo, Member, IEEE, M. Aquilano, M. Bonaccorsi, I. Mannari, M.C. Carrozza, Member, IEEE, \\ P. Dario, Member, IEEE
}

\begin{abstract}
This paper aims to show the effectiveness of a (inter / multi)disciplinary team, based on the technology developers, elderly care organizations, and designers, in developing the ASTRO robotic system for domiciliary assistance to elderly people. The main issues presented in this work concern the improvement of robot's behavior by means of a smart sensor network able to share information with the robot for localization and navigation, and the design of the robot's appearance and functionalities by means of a substantial analysis of users' requirements and attitude to robotic technology to improve acceptability and usability.
\end{abstract}

\section{INTRODUCTION}

$\mathrm{N}$ EW European population projections have recently underlined that the number of elderly persons living in Europe will quickly increase in the coming years [1]. This demographic trend will lead: (1) to a growing number of older people living alone and in need of (intensive) care, (2) to an ageing workforce and (3) to more financially wellappointed and wealthy senior citizens ready to enjoy their third age [2].

Nowadays the society sustains elderly people to age well by means of medical cures, socio-medical services, and other social activities but this request of support and assistance is so high that it is becoming difficult to manage and delivery. Robotics and ICT technologies represents nowadays a profitable solution for more efficient management and delivery of health and social care, as well as improvement of independent living, quality of life and efficient cares of elderly people.

Although a great deal of knowledge has been gained in industrial robotics, this technology is not completely ready to transfer into the assistive field. The reason is firstly due to the fact that, if from one hand industrial systems concentrate

Manuscript received April 15, 2011. This work was supported by the ASTROBOBILE project, an Experiment funded in the context of the ECHORD project.

F. Cavallo is with the BioRobotics Institute of Scuola Superiore Sant'Anna, Pisa, Italy (corresponding author to provide phone: +39050883474; fax: +39050883101; e-mail: f.cavallo@ sssup.it).

M. Aquilano is with the BioRobotics Institute of Scuola Superiore Sant'Anna, Pisa, Italy (e-mail: m.aquilano@ sssup.it).

M. Bonaccorsi is with the BioRobotics Institute of Scuola Superiore Sant'Anna, Pisa, Italy (e-mail: m.bonaccorsi@sssup.it).

I. Mannari is with the BioRobotics Institute of Scuola Superiore Sant'Anna, Pisa, Italy (e-mail: i.mannari@sssup.it).

M.C. Carrozza is with the BioRobotics Institute of Scuola Superiore Sant'Anna, Pisa, Italy (e-mail: carrozza@ sssup.it).

P. Dario is with the BioRobotics Institute of Scuola Superiore Sant'Anna, Pisa, Italy (e-mail: p.dario@ sssup.it). on relatively low task flexibility with high precision, accuracy and repetition in highly structured environments, on the other hand domestic systems concentrate on high task flexibility and adaptability with low precision and accuracy in largely unstructured environments [3]. Secondly, many robotic designers attempted to adapt existing engineering solutions, designed for other purposes, to barely defined health-care problems [4]. Thirdly, another reason is that the aim is not to replace an existing human or a human task, as is often the case with industrial robotics, but rather to support and enhance the user's abilities, which is a much more complex situation and means that careful balances must be struck between the roles of users and devices [5] Fourthly, because of end-user needs are often much more complex than those for industrial tasks, technologists and engineers always concentrate on technical product design, ignoring social and human aspects, and also acceptability and usability issues [6]. Fifthly, the heterogeneity of older people and the diversity of their living circumstances lead to a difficult customization of robotic systems [5]. Current research has shown that acceptance of assistive technology will require much more attention to users' preferences [7-8]. In this context, quality is defined in terms of efficiency, reliability, robustness, simplicity, safety, and cost.

This paper revolves around all these themes and aims to demonstrate the necessity and effectiveness of a (inter / multi)disciplinary team, i.e. technology developers, end-user organizations and designers, in developing a robotic system for domiciliary assistance to elderly people (ASTROMOBILE project Oct.2010 - Mar.2012).

\section{ASTROMOBILE PROJECT}

The ASTROMOBILE project (an Experiment of the ECHORD project) focuses on the development and experimentation of a robotic assistive platform integrated in smart environments, able to provide basic services of daily life with advanced and natural interaction with users. The design and implementation of ASTRO robot is sustained by a multidisciplinary team in which technology developers, designers and end-user representatives are collaborating using a user-centred design approach. The mobile robotic platform used for the ASTROMOBILE project is the commercial SCITOS-G5 (Metralabs GmbH, Germany) [9].

\section{A. Main developmental aspects}

The main issues of ASTROMOBILE project concern: 
1. the improvement of ASTRO's behaviour by means of a smart sensor network able to share information with the robot (Ubiquitous Robotics);

2. the design of ASTRO's appearance and functionalities by means of a substantial analysis of users' requirements and attitude to robotic technology to improve acceptability and usability;

3. the design of an advanced human robot interface based on natural language and able to enhance the usability and interaction of elderly users with the ASTRO robot (this point will be explained in a future work).

\section{B. Developmental methodology}

The methodology used in ASTROMOBILE foresees the involvement of a multidisciplinary team consisted of engineers, therapists, clinicians, psychologists, designers, and end-users, which strictly collaborate in order to identify real needs of users and caregivers and to develop the most suitable and appropriate technological solution. The approach includes precise work steps: first a robust analysis of the end-users' requirements described in the current literature to have an idea of the main functionalities of the robot; then a well structured program of interviews, focus groups and workshops with end-users and caregivers to point out end-users' needs considering life styles, habits, needs, quality of life, pathologies, cognitive and/or motor deficits, etc. A specific validation protocol is also conceived to verify functionalities in order to test the usability and its effectiveness in relation to features and opinions of caregivers. Finally a crucial step is a repetitive experimentation of the robot with real user in identified scenarios (see next paragraph) in order to identify possible weaknesses and to improve the performance of the robot.

\section{Scenarios and functionalities}

The ASTRO robot was conceived to accomplish some specific tasks in typical domestic scenarios where elderly people spend most of their daily life. The definition of these scenarios and functionalities comes from the previous experience of authors in assistive and robotic technologies [10-11] and the possible functionalities easily implementable on the SCITOS-G5. The functionalities of the ASTRO robot can be provided in two ways:

1) On-demand, when the users request by themselves to the robot to provide services:

$>$ The user calls the robot everywhere in the home and can ask for some services, such as help request, drugs request, assistance request, Skype call, status of the environment, etc.

$>$ Caregivers ask to remotely monitor situations by tele-operated features (camera on the robot).

2) Autonomously, when the robot pro-actively provides services to the users:

$>$ The robot goes to the user to communicate something, such as reminding, warning, etc.

$>$ The robot alerts for critical situations in the environment, such as door open, faucet, gas, etc.
$>$ The robot reminds for appointments or deadlines based on a scheduled database, such as take drugs, etc.

\section{INTEGRATION IN SMART ENVIRONMENTS}

The ASTRO robot is conceived to be integrated in a smart environment infrastructure in order to improve its behaviour. In particular a smart sensor network was used to enhance the localization and navigation capabilities of the robot, in order to perform high quality and acceptable services for users. The interaction between robot and environment by means of the pervasive sensor network will led to a sort of awareness, thanks to which the robot will exactly know the position of users, itself and objects to reach in the workspace.

\section{A. Smart sensor network}

The wireless sensor network is conceived to accomplish two aspects: to make the environment aware of what happen in indoor spaces and to work as an "internal GPS". This network, based on ZigBee technology (SPZB250 boards from ST Microelectronics), is made of various sensor nodes, some of them placed in fixed positions as anchors in the environment, some mobile nodes set on the robot and on the users and other nodes placed on objects, furniture and appliances. In the context of ASTROMOBILE project the sensor network is used to improve the indoor localization capabilities: the fixed anchors will communicate with mobile nodes and, by measuring the RSSI signal for each communication, the position of mobile nodes will be computed inside the environment [12-17]. This allows a sort of awareness, thanks to which the robot will exactly know the position of users or objects to reach in the workspace, improving the quality of services. In order to develop a reliable and efficient RSSI-based localization algorithm, different mathematical models and typology of antennas have been preliminary tested.

\section{B. Models for distance estimation}

The distance estimation between two nodes using the RSSI value was performed comparing three different models:

1) the HATA model in semi-logarithm coordinates

$$
\begin{aligned}
& \mathrm{P}(\mathrm{d})=\mathrm{P}_{0}-\mathrm{n}_{\mathrm{p}} \cdot \mathrm{X} \\
& \mathrm{X}=10 \cdot \log \left(\frac{\mathrm{d}}{\mathrm{d}_{0}}\right)
\end{aligned}
$$

where $P_{0}$ and $n_{p}$ are respectively the intercept and the slope of the linear regression of RSSI values $(P(d))$ vs. distances $(d)$;

2) the HATA model with empirical calculation of RSSI0 at reference distance of $1 \mathrm{~m}$,

$$
P(d)=P\left(d_{0}\right)-10 \cdot n_{p} \cdot \log \left(\frac{d}{d_{0}}\right)
$$

where $P\left(d_{0}\right)$ is measured RSSI value at distance $d_{0}(1 \mathrm{~m})$ and $n_{p}$ is the path loss;

3) the linear model

$$
P(d)=m \cdot d+q
$$


where $m$ and $q$ are respectively the intercept and the slope of the linear regression of RSSI values $(P(d))$ vs. distances $(d)$.
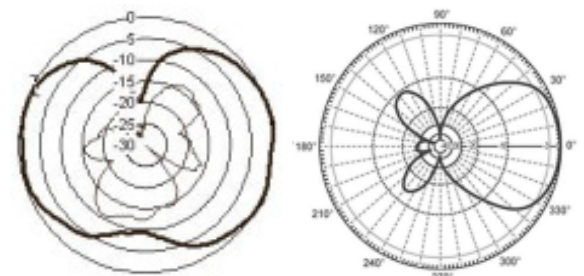

Fig. 1. Horizontal polar diagrams of antennas with omni-directional (left) and directive (right) emission.

Further three typologies of antennas were used: omnidirectional linear polarization, directive linear polarization and directive circular polarization (Fig. 1).

\section{Results}

Preliminary experiments were performed for 1D localization setup. The robot was instructed to move along a straight line between two anchor nodes, placed at a distance of $3 \mathrm{~m}$ and height of $0.6 \mathrm{~m} \mathrm{[18-19].} \mathrm{The} \mathrm{Vicon} \mathrm{optical} \mathrm{localization}$ system (http://www.vicon.com) was used to validated the measured localization of the robot with respect to anchor nodes.

Localization data were acquired for three different configurations:

1) anchor and mobile nodes with linear polarization / omni directional directivity antennas (Linear configuration);

2) anchor and mobile nodes with circular polarization / directive polar diagram antennas (Circular configuration);

3) anchor nodes with circular polarization / directive polar diagram antennas and mobile node with linear polarization / omni directional directivity antenna (Hybrid configuration)

For each configuration, ten trials were acquired, measuring the RSSI value with the SPZB250 boards and the true position with the Vicon system, and the three models were evaluated (Fig. 2). Estimating the RMSE for each configuration and model, the best results were achieved with the linear model, which we realized that it is more suitable for indoor environments (Table I).

\section{ACCEPTABILITY AND USABILITY}

The study on the design of the robot's appearance and on
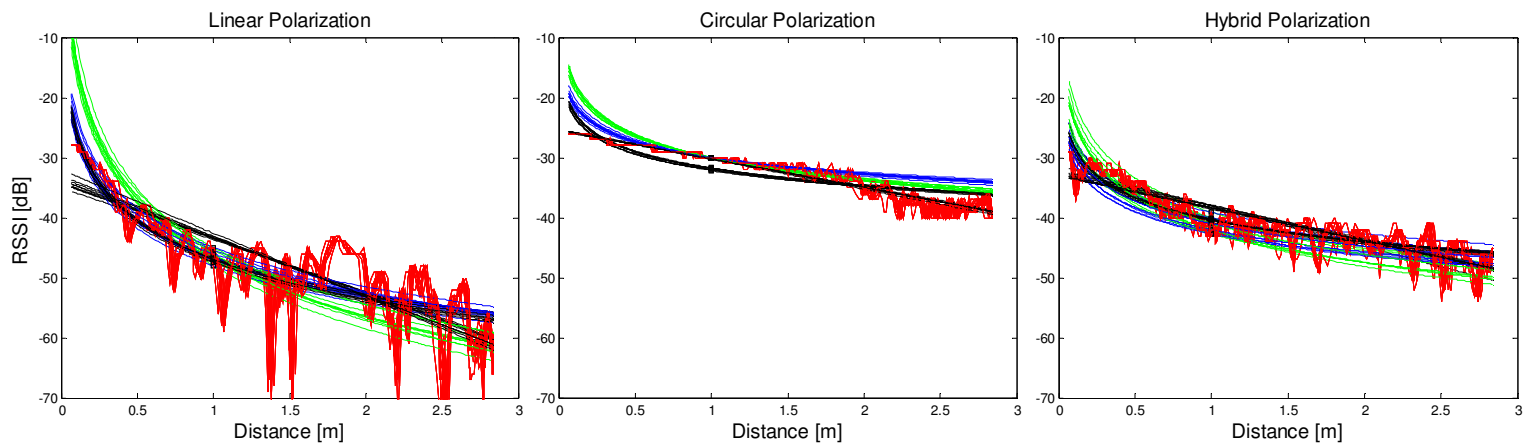

Fig. 2. Results for linear (left), circular (middle) and hybrid (right) polarization configuration. Red line is the RSSI values acquired with the SPZB250 board. Green, blue and black lines are respectively the HATA model in semi-logarithm coordinates, the HATA model with empirical calculation of RSSI0 at reference distance of $1 \mathrm{~m}$, and the linear model. 

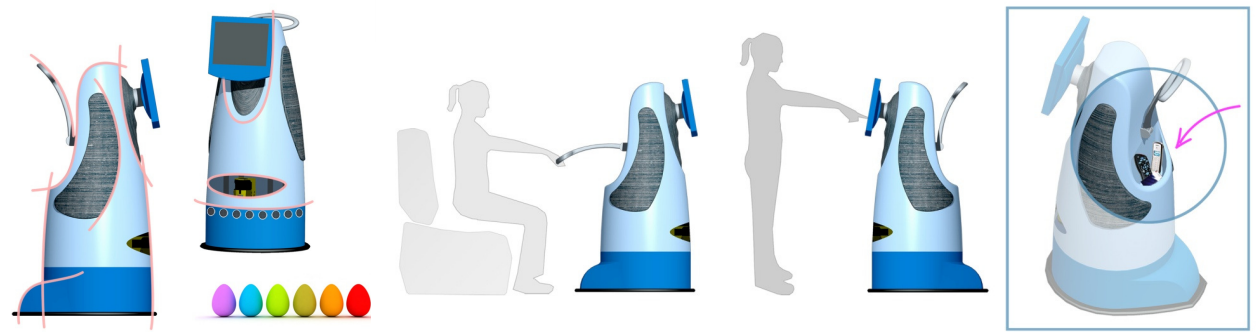

Fig. 3. From left to right: aesthetic and friendliness of ASTRO with smooth lines and comfortable colors and safety with the usage of soft materials; affordability and usability of ASTRO in using the handle bar and in interacting with the touch screen; usefulness in placing object in the glove box.

\section{B. Usefulness}

Usefulness is the propriety of the robot to improve the quality of life of users by means of concrete functionalities. To make the ASTRO robot really useful for elderly users as well as its "active" services, ASTRO robot was characterized by the presence of a handle bar to help users to lean on or stand up, a glove box to carry small objects (i.e. drugs, handkerchiefs, mobile phone, keys, remote control, etc.), and an adjustable touch screen to interact easily with the robot.

\section{Affordance}

Affordance is the propriety of the robot to make evident its functionalities. So the users, looking at the robot, can immediately understand what the robot is used for.

In ASTRO robot the handle bar and glove box were designed to be ergonomic and to immediately show their functionalities and how they can be used. The touch screen was designed as the head of the robot in order to be easily used as the communication part of the robot.

Furthermore to comply with this issue, the ASTRO robot's cover holds LEDs that change their color on the base of the status or functionalities that they are providing. The association of colors to the services was chosen considering natural and commonly used relations, for example the orange is associated to a context of warning and the red to an alert related to dangers.

\section{CONCLUSIONS}

In this paper we presented the first multidisciplinary design phase of ASTROMOBILE, in which a sensor network was used to enhance the localization and navigation capabilities and a centered design approach was followed for satisfy acceptability and usability requirements.

Even if localization and navigation capabilities were investigated only in 1D environments, however this permitted us to preliminary characterize and calibrate the system for different antennas and models. Results clearly demonstrate that the circular configuration and the linear model is the best combination with the lowest RMSE in 1D environments. Experiments in 2D workspaces have already been performed but results analysis is still in progress.

Several properties of the robot for analyzing the acceptability and usability were considered. Next steps include the close contact with end-users, whose opinions and requirement analysis are fundamental to assess and validate such preliminary design aspects, before the true production of the first prototype.

\section{REFERENCES}

[1] “The Global Aging Experience Project. Ethnographic Research", Intel research, 2010.

[2] G. van der Broek, F. Cavallo, and C. Wehrmann, "AALIANCE Ambient Assisted Living Roadmap", Vol. 6, IOS Press, 2010.

[3] Q. Meng, M.H. Lee, "Design issues for assistive robotics for the elderly", Advanced Engineering Informatics 20 (2006) 171-186.

[4] Fehr L, Langbein WE, Skaar SB, "Adequacy of power wheelchair control interfaces for persons with severe disabilities: a clinical survey", Rehabil Res Dev 2000; 37(3).

[5] McCreadie C, Tinker AA, "The acceptability of assistive technology to older people", Ageing Soc 2005; 91-110.

[6] Sixsmith A, Sixsmith J., "Smart technologies: meeting whose needs?" Telemed Telecare 2000; 190-2.

[7] Cowan D, Turner-Smith A., "The role of assistive technology in alternative models of care for older people", With respect to old age, Vol.2, Centre of Rehab. Engineering, King's College London, 1999.

[8] Ya-Huei Wu, Christine Fassert, Anne-Sophie Rigaud, "Designing robots for the elderly: Appearance issue and beyond", Archives of Gerontology and Geriatrics (2011), in press.

[9] Metralabs website: http://www.metralabs.com/

[10] P. Dario, E. Guglielmelli, C. Laschi, and G. Teti "MOVAID: a personal robot in everyday life of disabled and elderly people", Technology and Disability, vol. 10, 1999, pp. 77-93.

[11] P. Salvini, A. Armato, I. Mannari, B. Mazzolai, C. Laschi, P. Dario, "A Methodology for Designing Acceptability in Relation to a Service Robot Physical Appearance", 8th International Workshop on Humanfriendly Welfare Robotic Systems, 21-23 October 2007.

[12] M. Hata, "Empirical Formula for Propagation Loss in Land Mobile Radio Services", IEEE Trans. On vehicular technology, VOL. VT-29, NO. 3, August 1980.

[13] Y. Rahal, H. Pigot, P. Mabilleau, "Location estimation in a smart home: System implementation and evaluation using experimental data", International J. of Telemedicine and Applications, Vol.2008.

[14] J. A. Dabin, N. Ni, A. M. Haimovich, E. Niver, H. Grebel, ”The effect of antenna directivity on path loss and multipath propagation in UWB indoor wireless channel", IEEE Conference on Ultra Wideband Systems and Technologies, 2003.

[15] Dimitrios Lymberopoulos, Quentin Lindsey, and Andreas Savvides, "An Empirical Characterization of Radio Signal Strength Variability in 3-D IEEE 802.15.4 Networks Using Monopole Antennas", EWSN 2006, LNCS 3868, pp. 326-341.

[16] S. Hara, D. Zhao, K. Yanagihara, J. Taketsugu, K. Fuku, S. Fukunaga, K., "Propagation characteristics of IEEE 802.15.4 radio signal and their application for location estimation", 2005 IEEE 61st Vehicular Technology Conference, 2005.

[17] Angela Song-Ie Noh, Woong Jae Lee, Jin Young Ye, "Comparison of the mechanisms of the ZigBee's indoor localization algorithm", 9th ACIS International Conference on Software Engineering, Artificial Intelligence, Networking and Parallele/Distribuited Computing; IEEE Computer Society 2008; Pages 13 - 18.

[18] F. Cavallo, M. Aquilano, M. Bonaccorsi, M. C. Carrozza, P. Dario, "Preliminary characterization of an indoor localization system using a ZigBee based sensor network and a triaxial accelerometer," AALIANCE Conference, Malaga, Spain, March 2010.

[19] P. Nepa, F. Cavallo, M. Bonaccorsi, M. Aquilano, M.C. Carrozza, P. Dario, "Experimental Analysis of RSSI-Based Indoor Location Systems with WLAN Circularly Polarized Antennas", MobiHealth 2010, pp 1-8, Cipro,2010. 